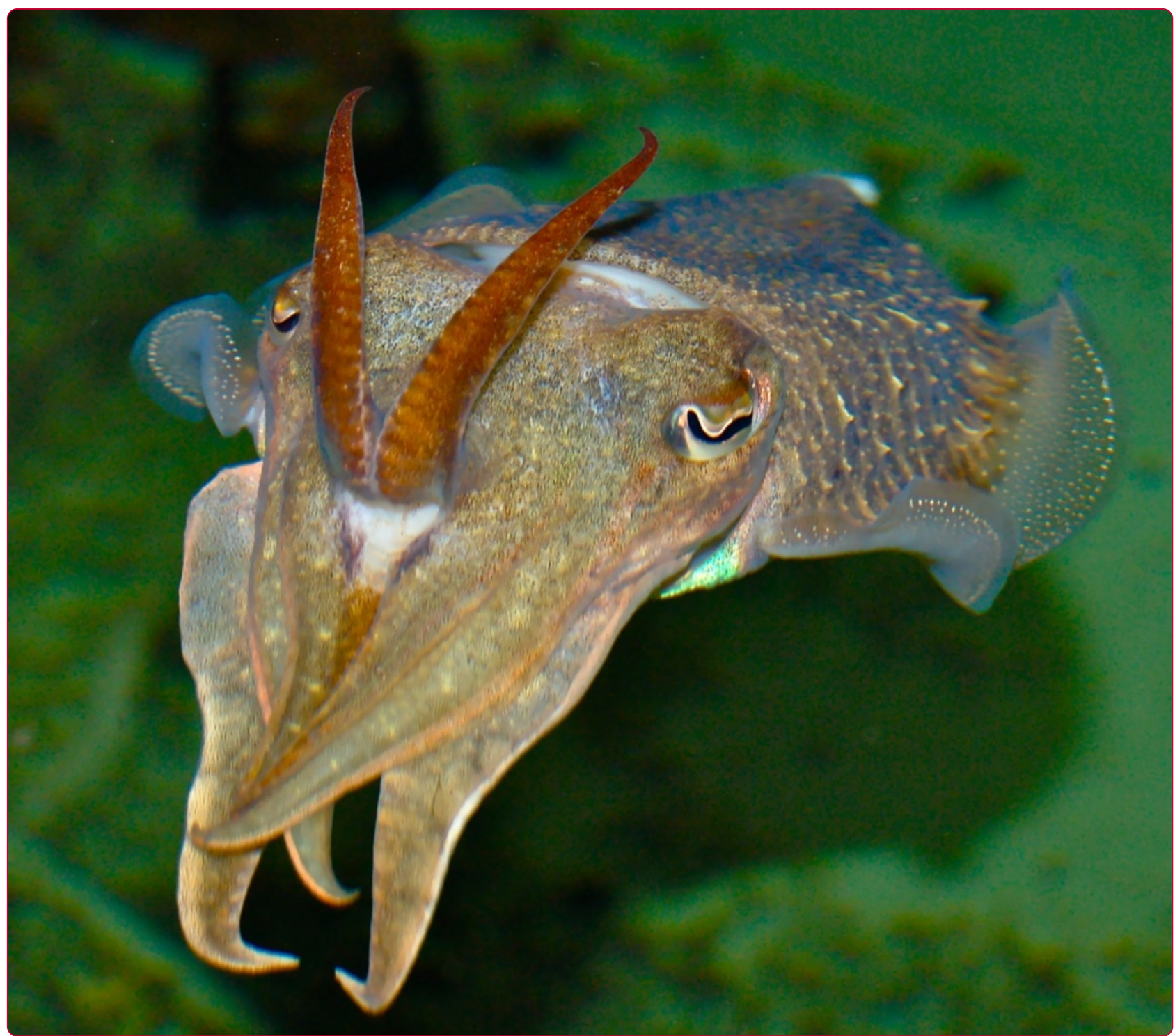

A new haemocyanin in cuttlefish (Sepia officinalis) eggs: sequence analysis and relevance during ontogeny

Thonig et al. 


\title{
A new haemocyanin in cuttlefish (Sepia officinalis) eggs: sequence analysis and relevance during ontogeny
}

\author{
Anne Thonig ${ }^{1}$, Michael Oellermann ${ }^{1}$, Bernhard Lieb² and Felix Christopher Mark ${ }^{1 *}$
}

\begin{abstract}
Background: Haemocyanin is the respiratory protein of most of the Mollusca. In cephalopods and gastropods at least two distinct isoforms are differentially expressed. However, their physiological purpose is unknown. For the common cuttlefish Sepia officinalis, three isoforms are known so far, whereas for only two of them the complete mRNA sequences are available. In this study, we sequenced the complete mRNA of the third haemocyanin isoform and measured the relative expression of all three isoforms during embryogenesis to reveal a potential ontogenetic relevance.

Results: The cDNA of isoform 3 clearly correlates to the known Sepia officinalis haemocyanin subunits consisting of eight functional units and an internal duplicated functional unit d. Our molecular phylogenetic analyses reveal the third isoform representing a potentially ancestral haemocyanin isoform, and the analyses of the expression of haemocyanin type 3 reveal that haemocyanin type 3 only can be observed within eggs and during early development. Isoforms 1 and 2 are absent at these stages. After hatching, isoform 3 is downregulated, and isoform 1 and 2 are upregulated.
\end{abstract}

Conclusions: Our study clearly shows an embryonic relevance of the third isoform, which will be further discussed in the light of the changes in the physiological function of haemocyanin during ontogeny. Taken together with the fact that it could also be the isoform closest related to the common ancestor of cuttlefish haemocyanin, the phylogeny of cuttlefish haemocyanin may be recapitulated during its ontogeny.

Keywords: Respiratory protein, Cephalopods, Mollusks, Embryogenesis, Haematopoiesis, Development

\section{Background}

Haemocyanin is the respiratory protein of arthropods and molluscs. This copper-containing pigment occurs freely dissolved in the haemolymph of these animals and is coloured blue when oxygenated. The haemocyanins of both mentioned phyla have a copper type-3 oxygenbinding site, but otherwise show structural differences and evolved separately [1-3]. The basal quaternary structure of molluscan haemocyanins is a cylindrical decamer composed of $\sim 350-400 \mathrm{kDa}$ large subunits (SU) [4]. In Polyplacophora and Cephalopoda, only decamers can be observed floating in the haemolymph [5,6]. In Bivalvia and Gastropoda, they are also able to form didecamers

\footnotetext{
* Correspondence: fmark@awi.de

${ }^{1}$ Integrative Ecophysiology, Alfred Wegener Institute for Polar and Marine Research, 27570 Bremerhaven, Germany

Full list of author information is available at the end of the article
}

and multidecamers $[7,8]$. The single subunits usually consist of 7-8 tandemly arranged functional units (FU) of $\sim 50 \mathrm{kDa}$ each. Every FU exhibits one oxygen-binding site [1]. These paralogous FUs evolved by subsequent gene/exon duplications and fusions $[9,10]$. The subunits of cephalopod haemocyanins are characterised by the lack of the most C-terminal FUh $[6,10]$. The $350 \mathrm{kDa}$ haemocyanin subunits of Tetrabranchiata and Octobrachia consist of 7 FUs (a-g), contrary to the 8 FUs ( 400 kDA) in Decabrachia $[1,11]$. For the common cuttlefish (Sepia officinalis) and Spirula (Spirula spirula), it has been shown that a duplication of FUd led to the 8 FUs (a-b-c-d-d'-e-f-g; $[12,13])$.

Besides the duplications of single FUs, duplications of the complete haemocyanin gene occurred as well. This happened several times independently in different groups and resulted in different isoforms of the protein [4]. 
Gastropods and bivalves can feature one or two isoforms $[1,8,14-20]$, while in cephalopods probably only the tetrabranchiate Nautilus pompilius is restricted to a single haemocyanin [6]. The recent coleoid cephalopods were all found to express at least two different isoforms of the respiratory protein (e.g., Sepia officinalis, DeGeest et al., unpubl.; Allotheuthis media, Oellermann et al., unpubl.; E. dofleini [9]). So far, the complete mRNA sequences of haemocyanin isoform 1 (SoH1 [GenBank: DQ388569]) and isoform 2 (SoH2 [GenBank:DQ388570]) have been identified in Sepia officinalis. Next to these two known isoforms there is great evidence for a third one in the cuttlefish (SoH3; [21,22]), whereof one fragment of $\sim 1,000$ bp has already been sequenced [GenBank: JN392726]. The existence of these isoforms raises questions about their evolution referring to origin and structural changes as well as to their physiological relevance.

Due to its moderate evolutionary rate, haemocyanin has been used to estimate the divergence time of different groups among Mollusca, e.g., the most recent common ancestor of cephalopods, Dibranchiata and Decabrachia was calculated [13]. Additionally, the duplication of the $S$. officinalis isoforms could be dated to $\sim 90$ million years ago (Mya), whereas the isoforms of $E$. dofleini split much more recently. In both isoforms of S. officinalis-as well as in S. spirula-the duplicated FUd is present $[11,13]$. This points to a duplication event of FUd earlier than the split of the isoforms. Yet with no complete sequence of the third isoform, no further implications concerning the origin and evolutionary development of that isoform can be drawn.

However, all isoforms are expressed, and SoH1 and SoH2 even display different theoretical isoelectric points (in silico: SoH1: $\mathrm{pI}=5.79$, SoH2: $\mathrm{pI}=5.85$ ), which in turn could affect the oxygen affinity $[21,22]$. The existence of different isoforms might therefore serve a physiological purpose. Indeed, it has already been shown that some vertebrates and invertebrates can change the composition or concentration of their respiratory protein in response to changing environmental parameters such as temperature and oxygen partial pressure $\mathrm{PO}_{2}$ (Gadus morhua [23], Daphnia magna and D. pulex [24,25]; Triops longicaudatus [26], Artemia franciscana [27]). Moreover, cephalopods display the highest metabolic rates among invertebrates and therefore have to rely on an efficient oxygen supply, which is also indicated by the great Bohr effect of their haemocyanin [28-30]. Nevertheless, S. officinalis displays no difference in relative expression of the haemocyanin isoforms in response to changing temperature, hypercapnia or hypoxia [22, Thonig et al., unpublished].

Besides adaptation to changing environmental conditions, different isoforms of respiratory proteins are also known to be expressed during the course of ontogeny (Crustaceans [31], Cancer magister and C. productus [32-35], Artemia salina [36], Schistocerca americana [37], Chironomus plumosus and Chironomus thummi [38,39], chicken [40] and mammals [41]). For example, humans start with the expression of embryonic haemoglobin, which is replaced by foetal, and afterwards by adult haemoglobin (e.g., [42]). Likewise, the maternal haemocyanin in the oocytes of the crustacean Cancer magister is subsequently replaced by embryonic, juvenile and adult haemocyanin [43].

Thus, the evolution of embryonic versions of the respective respiratory proteins (haemoglobin and haemocyanin) occurred independently in invertebrates and vertebrates. This might indicate common challenges referring to oxygen supply during early development. Therefore, an ontogenetic relevance of the different haemocyanin isoforms could be the case for Sepia officinalis. This is assumable especially as cuttlefish embryos have to face hypoxic and hypercapnic conditions towards the end of embryogenesis since their egg capsule functions as a diffusion barrier [44-46]. Actually, the existence of an embryonic haemocyanin in cuttlefish had already been proposed on the basis of electrophoretic distinct protein fractions of haemocyanin in embryos, juveniles and adults of Sepia officinalis $[47,48]$. Furthermore, Beuerlein et al. [49] identified haemocyanin mRNA [GenBank:AF401231] not only in the branchial glands of cuttlefish embryos, which would reflect the situation in adults, but also in haemocytes, the branchial hearts, the midgut gland and the renal appendages. This indicates both a switch of function in the haemocyanin metabolism of these tissues accompanied with hatching and a change of the site of synthesis of haemocyanin during ontogeny [50]. This would be well in line with a shift of haemocyanin expression. Finally, Strobel et al. [22] detected the highest relative expression of the recently found potential isoform 3 in newly hatched cuttlefish, while it decreased significantly with time after hatching. This could point out an embryonic function of haemocyanin isoform 3 .

We therefore identified the complete sequence of haemocyanin isoform 3 , and revealed its origin and evolutionary history. On the other hand, we tried to elucidate the role of this isoform 3 during embryogenesis by analysing the relative expression of the haemocyanin isoforms in different embryonic stages of the cuttlefish Sepia officinalis; in this way, we combined an evolutionary and physiological approach to characterise the new differentially expressed haemocyanin isoform 3 of S. officinalis.

\section{Methods}

\section{Animal collection and sampling}

Clusters of cuttlefish eggs were collected in May 2011 in Chioggia (Adria, Italy) and transferred to and kept at the 
Alfred Wegener Institute in Bremerhaven, Germany. For further investigations, the egg capsules were removed, and the length and width of the embryos as well as their developmental stages according to Lemaire [51] were determined. We distinguished five different stages: E1 (Lemaire 21-24), E2 (Lemaire 25-26), E3 (Lemaire 26-27), E4 (Lemaire 28-29) and H (Lemaire 30). Hereafter, either the complete embryos, the gills or the outer yolk sac were immediately frozen in liquid nitrogen for subsequent RNA analysis. Additionally, adult specimens collected in the English Channel (Caen) in 2010 and raised at the Alfred Wegener Institute at $15^{\circ} \mathrm{C}$ were used to compare the expression of haemocyanin mRNA (Thonig et al., unpublished).

\section{cDNA synthesis}

Due to the low weight of the individual embryonic and tissue samples, RNA was mainly extracted from pools of different individuals of the same developmental stages using the RNeasy Mini Kit (Qiagen GmbH, Hilden, Germany). The pools ranged from one to eight individuals corresponding to 9 to $102 \mathrm{mg}$ input material (Table 1). The samples were homogenised for $15 \mathrm{~s}$ at 6,500 rpm in a Precellys beadmill (Bertin Technologies, Peqlab Biotechnologies $\mathrm{GmbH}$, Erlangen, Germany) with doubled amount of recommended lysis buffer RLT (Qiagen GmbH, Hilden, Germany) at room temperature. Further extraction procedures followed the RNeasy Mini Kit protocol. A DNase treatment of each extract was carried out according to the Ambion Turbo DNA-free ${ }^{\mathrm{TM}}$

Table 1 Tissue and embryo pools of S. officinalis used for CDNA synthesis and subsequent real-time qPCR

\begin{tabular}{|c|c|c|c|c|c|}
\hline Name & $\begin{array}{c}\text { Stage } \\
\text { (Lemaire) }\end{array}$ & Tissue & $\begin{array}{c}\text { No. of } \\
\text { individuals }\end{array}$ & $\begin{array}{c}\text { Input } \\
\text { material [mg] }\end{array}$ & Comment \\
\hline $\mathrm{E} 1_{-} \mathrm{Y}$ & $21-23$ & Yolk & 2 & 102 & No statistics \\
\hline E1_E1 & $21-23$ & Embryo & 8 & 49 & \\
\hline E1_E2 & $21-23$ & Embryo & 2 & 13 & \\
\hline E2_E1 & $24-26$ & Embryo & 3 & 45 & \\
\hline E2_E2 & $24-26$ & Embryo & 2 & 25 & \\
\hline E2_E3 & $24-26$ & Embryo & 2 & 28 & \\
\hline E3_E1 & $26-27$ & Embryo & 2 & 43 & \\
\hline E3_E2 & $26-27$ & Embryo & 2 & 45 & \\
\hline E3_E3 & $26-27$ & Embryo & 1 & 29 & \\
\hline E3_E4 & $26-27$ & Embryo & 1 & 34 & \\
\hline E3_E5 & $26-27$ & Embryo & 2 & 48 & \\
\hline E3_E6 & $26-27$ & Embryo & 1 & 31 & \\
\hline E4_E1 & $28-29$ & Embryo & 2 & 19 & \\
\hline E4_E2 & $28-29$ & Embryo & 1 & 47 & \\
\hline E4_E3 & $28-29$ & Embryo & 1 & 32 & \\
\hline H_G & 30 & Gill & 4 & 12 & No statistics \\
\hline
\end{tabular}

Kit (Ambion, Applied Biosystems, Darmstadt, Germany). Afterwards, cDNA synthesis was performed via RT-PCR with $0.4 \mu \mathrm{g}$ of RNA either according to the High Capacity cDNA Reverse Transcription Kit (Applied Biosystems, Darmstadt, Germany) for Real-Time PCR or according to the Invitrogen Superscript III cDNA synthesis kit (Life Technologies) for subsequent sequencing of the haemocyanin isoform 3 cDNA.

\section{qPCR and statistical analysis}

The relative expression of the different haemocyanin isoforms was analysed using an ABI 7500 Real-Time PCR System with the respective 7500 SDS Software, version 1.3 (Applied Biosystems, Darmstadt, Germany). The $20 \mu \mathrm{l}$ PCR reaction was performed in MicroAmp ${ }^{\circ}$ Optical 96 well plates (Applied Biosystems, Darmstadt, Germany) using the SYBR ${ }^{\circ}$ Green PCR Master Mix (Applied Biosystems, Germany). Each qPCR replicate contained 2 ng of cDNA and isoform-specific primers with a final concentration of $300 \mathrm{nM}$. The primers were designed and optimised by Strobel et al. [22] on the basis of the respective sequences available in GenBank:

\section{Isoform 1 [GenBank:DQ388569]: \\ SofHc1_fwd CTTTTCGAGTTTACCAGCTCTTGTT, SofHc1_rev CCTGCAACGTCAATATATGAGTGAT. Isoform 2 [GenBank:DQ388570]: \\ SofHc2_fwd TCTCCCGTTTTTGGTAACTGAAC, SofHc2_rev TGTCAGCAACATCAATATAACCATGA. Isoform 3 [GenBank:JN392726]: \\ SofHc3_fwd TGCTCCGTCAACCAATGTCC, SofHc3_rev TCCAGGGCATCTCGTTTTCAC.}

The PCR programme was composed of a $10 \mathrm{~min}$ activation step at $95^{\circ} \mathrm{C}$ and 40 amplification cycles $(15 \mathrm{~s}$ at $95^{\circ} \mathrm{C}$ for denaturing, $1 \mathrm{~min}$ at $60^{\circ} \mathrm{C}$ for annealing and elongation) with a single fluorescence measurement during the annealing step. A melting curve analysis was performed to ensure the amplification of one single product. Efficiencies for the different primer pairs were calculated according to the equation $\mathrm{E}=10^{(-1 / \text { slope })}$, using the decadic logarithm of cDNA input plotted against the cycle thresholds $\left(\mathrm{C}_{\mathrm{T}}\right)$ for five template dilution steps (efficiency SoH1: 1.843, SoH2: 1, 1.859, SoH3: 1.824 ).

For further analysis, the $\mathrm{C}_{\mathrm{T}}$-values were transformed into relative quantities [52] using MS Excel according to the following equation: quantity $(x)=$ efficiency ${ }_{\mathrm{T}}\left(\mathrm{C}_{\mathrm{T}}\right.$ (Minimum) $-\mathrm{C}_{\mathrm{T}}(\mathrm{x})$ ) . Furthermore, the relative quantities were normalised to adjust differences in template input using the normalisation coefficients calculated by geNorm [53]. For this reason, the potential housekeeping genes cytochrome c oxidase (COX) and nucleolin were analysed via Real-Time PCR as well. The respective primer pairs were designed on the basis of 454 pyrosequencing 
results of cDNA of the branchial gland of adult Sepia officinalis (Lieb \& Mark, unpublished) using Primer Express Software for Real-Time PCR, version 3.0 (Applied Biosystems, Darmstadt, Germany) (nucleolin: Nuc_fw CAAGCC GCAAAGAAAGACAAG, Nuc_rv GCTTTAGATTCCT TTTTGGAAGCA, COX: COX_fw CAATAGGAGCCGT TTTTGCG, COX_rv AGGGTAATCAGAGTATCGTCG TGG) and yielded efficiencies of 1.719 for COX and 1.809 for nucleolin. The relative quantities of the respective $C_{T}$ values of the potential housekeeping genes were then used to calculate the normalisation coefficients for the expression of the haemocyanin isoforms with geNorm.

Finally, Nalimov's test [54] on a significance level of 95\% was performed with the normalised relative quantities of the haemocyanin isoforms to remove outliers. The data were illustrated as the mean with standard error using GraphPad Prism 5.0b (GraphPad Software, Inc., La Jolla, CA, USA) and the expression trend using Adobe Illustrator (Adobe Systems Software Ireland Ltd., Ireland). Statistical analysis to determine significant differences in expression of the three haemocyanin isoforms during embryonic development was performed using the non-parametric Mann-Whitney U-Test via SPSS Statistics 19 (IBM, Ehningen, Germany).

\section{Sequencing}

The SoH3 cDNA sequence was obtained via primer walking using cDNA derived from yolk and embryonic samples of the earliest developmental stage as template to ensure a large amount of SoH3 mRNA. Touchdown PCRs were performed with one degenerated primer coding for the conserved copper-binding sites [10] and one SoH3-specific primer designed with MacVector 12.0 (MacVector, Inc., Cambridge, UK) based on the already known fragment of SoH3. The touchdown PCR protocols consisted of a $4 \mathrm{~min}$ activation step at $94^{\circ} \mathrm{C}, 12$ touchdown cycles with the annealing temperature decreasing by $1^{\circ} \mathrm{C}$ per cycle and finally 35 amplification cycles. The annealing temperature and elongation time of the PCR protocols were chosen with respect to the melting temperature of the primer pair and the length of the expected PCR product. The amplicons were separated in a $1.3 \%$ agarose gel with GelRed and extracted using the QIAQuick Gel Extraction Kit (Qiagen $\mathrm{GmbH}$, Hilden, Germany). Afterwards, the extracts were either used for an additional amplification and purification step to increase product concentration or sequenced directly by Eurofins MWG GmbH (Martinsried, Germany).

Additionally, PCR products were obtained using two degenerated primers [10]. Those ones and PCR products giving double peaks after the sequencing reactions were cloned into the pGemT-easy vector (Promega, Madison, WI, USA) or TOPO TA vector (Life Technologies Invitrogen, Darmstadt, Germany). A PCR with the standard vector primer M13 uni and M13 rev was followed by an RFLP analysis using several restriction enzymes (MunI, HinfI, AluI, SpnI, MboI, RsaI) to determine different inserts. These inserts were then purified using the QIAQuick Gel Extraction Kit and sequenced by Eurofins MWG GmbH (Martinsried, Germany) or GATC Biotech AG (Konstanz, Germany).

The 5' -end and $3^{\prime}$ - end of the SoH3 mRNA were amplified with the Ambion FirstChoice ${ }^{\circ}$ RLM-RACE Kit and the Invitrogen GeneRacer Kit (Life Technologies Invitrogen, Darmstadt, Germany) according to the manual and sequenced by Eurofins MWG GmbH (Martinsried, Germany) or GATC Biotech AG (Konstanz, Germany).

Additionally, genomic DNA was isolated from cuttlefish branchial glands using Qiagen DNeasy columns according to the manufacturer's protocol. After shearing of $1 \mu \mathrm{g}$ DNA (Covaris S2), an Illumina paired-end library was constructed according to TruSeq DNA Sample Preparation v2 protocol. First, the DNA was end repaired, 3'-ends were adenylated and adapters were ligated. Subsequently the DNA was amplified (10 cycles) and double size selected using the SPRIselect reagent kit (Beckman Coulter). The resulting DNA fragments ranged between 300 and 600 bp with a mean size of ca. 280 bp. Sequencing was done on 1.375 lanes of an Illumina HiSeq 2000 paired-end flowcell resulting in approximately $400,000,000$ reads. DNA processing and sequencing were done by Genterprise (Mainz).

\section{Sequence analysis}

The single sequences were trimmed, corrected on the basis of the chromatogram and assembled to a complete SoH3 sequence using Geneious 6.1.2 (Biomatters). Overlaps of at least $\sim 150 \mathrm{bp}$ were used to assure a correct assembly. The Illumina reads were trimmed and mapped on the cDNA sequence allowing maximum $10 \%$ mismatches per read. From this mapping the position of introns and the FUs could be deduced according to the GT/AG rule. The $5^{\prime}$ - and $3^{\prime}$-UTRs were annotated according to the derived open reading frame (ORF). The respective protein sequence was obtained via translation using the standard genetic code. Furthermore, a signal peptide was detected via SignalP v4.1 and potential $\mathrm{N}$-glycosylation sites using NetNGlyc1.0. Finally, the molecular weight as well as the theoretical isoelectric point of haemocyanin subunit 3 was determined via ExPASy Bioinformatics Resource Portal [55].

For phylogenetic analyses the haemocyanin sequences of Sepia officinalis (SoH1 [GenBank:DQ388569], SoH2 [GenBank:DQ388570]), Enteroctopus dofleini (A-type [GenBank:AY751301], G-type [GenBank:AF338426]), Nautilus pompilius [GenBank:AJ619741] and Haliotis tuberculata (HtH1 [GenBank:AJ252741], HtH2 [GenBank: AJ297475]) as outgroup were added from GenBank. For 
those sequences the FUs were annotated according to the genomic data of $S$. officinalis [6,10]. The multiple sequence alignments of the complete haemocyanin subunits and the FUd/d' were obtained using MUSCLE implemented in Geneious 6.1.2. The alignments were curated using Gblocks 0.91b ([56]; parameters used for haemocyanin/FUd alignment: minimum number of sequences for a conserved position: 5 , minimum number of sequences for a flanking position: $6 / 7$, maximum number of contiguous nonconserved positions: 8 , minimum length of a block: 10; allowed gap positions: none, use similarity matrices: yes) to eliminate poorly aligned positions. Subsequently, we determined the appropriate substitution model via ProtTest 3.2 [57] according to the corrected Akaike information criterion (AICc): WAG $+\mathrm{I}+\mathrm{G}+\mathrm{F}(\mathrm{I}=2.199$, $\mathrm{G}=0.199$ for complete $\mathrm{Hc}$ sequences), $\mathrm{WAG}+\mathrm{G}+\mathrm{F}$ $(\mathrm{G}=0.799$ for $\mathrm{FUd} / \mathrm{d}$ '). Based on these substitution models, Bayesian trees were calculated via MrBayes 3.2.1 [58] running an MCMC analysis with 2,000,000 generations and a sampling frequency of 200 generations. According to the respective trace files, the likelihoods had reached stationarity before $20 \%$ of the MCMC chain, which was therefore used as the burnin fraction. Additionally, maximum likelihood trees were calculated using the PhyML plugin of Geneious 6.1.2 [59] performing 1,000 replicates to calculate bootstrap values. Since the PhyML plugin did not allow to account for the amino acid frequencies $(+\mathrm{F})$, the following substitution models were chosen: $\mathrm{LG}+\mathrm{I}+\mathrm{G} \quad(\mathrm{I}=1.316, \mathrm{G}=0.144$; for complete Hc sequences), WAG $+\mathrm{I}+\mathrm{G} \quad(\mathrm{I}=0.193$, $\mathrm{G}=1.696$ for $\mathrm{FUd} / \mathrm{d}^{\prime}$ ).

A relaxed molecular clock was calculated with BEAST v1.5.4 using a Bayesian MCMC analysis [60]. The following settings were chosen: WAG $+\mathrm{I}+\mathrm{G}$ as substitution model, an uncorrelated log-normal distribution as clock mode and birth-death process as tree prior. Two analyses were run for 10,000,000 generations and were logged every 1,000 generation. Stationarity and appropriate mixing of each was checked using Tracer v1.5 [60]. The runs were combined using LogCombiner and the resulting file was processed with TreeAnnotator using a burnin of $20 \%$. An additional run only under the priors was performed to confirm that the chosen priors were sufficiently non informative. The split of GastropodaCephalopoda in the late Cambrian 550 50 Mya was used as calibration point $[6,7,17]$.

\section{Ethics statement}

All sampling of cuttlefish was conducted according to the ethics and guidelines of the German law and do not require a formal permit. The experiments have been communicated according to $\$ 8$ animal welfare act $(18.05 .2006 ; 8081$. I p. 1207) to the veterinary inspection office 'Senatorin für Arbeit, Frauen, Gesundheit,
Jugend und Soziales, Abt. Veterinärwesen, Lebensmittelsicherheit und Pflanzenschutz', Bahnhofsplatz 29, 28195 Bremen, Germany, evaluated and approved of on July 12, 2011.

\section{Results \\ Haemocyanin mRNA expression}

To investigate a potential ontogenetic relevance of the existence of different haemocyanin isoforms in cuttlefish, we measured their expression in different developmental stages of embryogenesis via Real-Time PCR. Thereby, we detected distinct differences during ontogeny referring to both the relative expression (Figure 1) and the ratio of the isoforms (Table 2). As shown in Figure 1, the mRNA of isoform 3 is highly expressed in embryos and also in the yolk sac of the earliest developmental stage. While SoH3 remains almost constantly at a high level of expression during embryonic development, significant amounts of SoH2 and SoH1 appear only during late organogenesis in stage E3 and E4, respectively. Though $\mathrm{SoH} 2$ could be detected in very low amounts already at stage E1 (even in the yolk sac) and E2, it only increased significantly during the following stages E3 and E4. Finally, SoH1 was first present at stage E3 with significantly increased expression shortly before hatching (E5). Thereby SoH3 represents about $99.8 \%-100 \%$ of the haemocyanin expressed in early organogenesis (E1 + E2) measured in the complete embryo (Table 2). Before hatching, SoH3 accounts for $66.6 \%$ of haemocyanin, while SoH2 represents $31.7 \%$ and SoH1 1.7\%. In gills of hatchlings $(\mathrm{H})$, ratios of the haemocyanin isoforms were measured as follows: SoH2 90.1\%, SoH1 9.7\% and SoH3 $0.3 \%$. This reflects the situation found in the branchial gland of adult cuttlefish (incubation at $15^{\circ} \mathrm{C}$ ): $\mathrm{SoH} 2$ 87.2\%, SoH1 12.8\% and SoH3 0.03\%.

\section{SoH3 sequence}

The SoH3 mRNA sequence (GenBank:KF306341) consists in total of $10,277 \mathrm{bp}$. It is composed of a $39 \mathrm{bp}$ 5 '-UTR, a 10,017 bp ORF containing the eight FUs and a 221 bp 3'-UTR. The typical AATAAA polyadenylation signal can be found 13 bp upstream of the poly-(A) tail. Partly different versions of the haemocyanin sequence were obtained from sequencing and could not definitely be assigned referring to either the chromatogram or genomic data, or via cloning. Therefore, 20 ambiguous sites of 10,277 bp in total (representing $0.2 \%$ ) remain in the sequence. They are mainly spread in the FUa (5), FUb (8) and FUc (7).

The deduced primary structure consists of 3,338 amino acids (aa), including a signal peptide of 26 aa and the actual polypeptide of 3,312 aa. This consists of 8 FUs ranging from 409 to 418 aa, reflecting the FUs found in the other known isoforms. Thus, the duplication of FUd/d' 


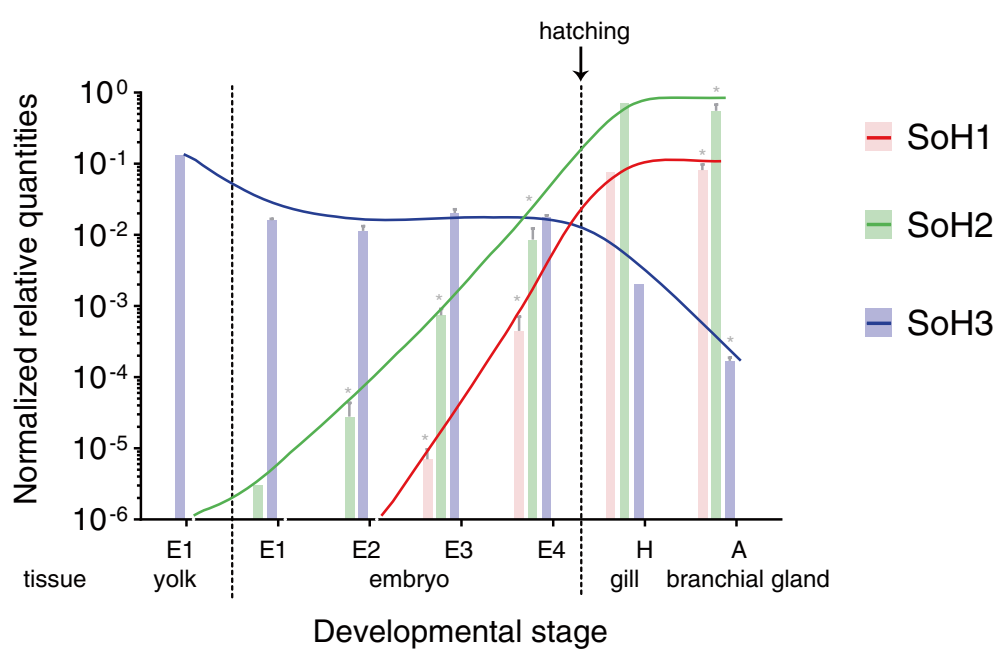

Figure 1 Haemocyanin expression during ontogeny. The mRNA expression of the different haemocyanin isoforms $(\mathrm{SoH} 1$, SoH2, SoH3) of Sepia officinalis in different tissues (yolk, embryo, gill, branchial gland) and developmental stages ranging from embryos (E1-E4) to hatchlings (H) and adults (A). The histogram shows the mRNA expression as normalised relative quantities with standard error. Thereby, asterisks indicate when the expression of the respective isoform differs significantly compared to the previous developmental stage on a significance level of 0.05 according to the Mann-Whitney-U test. Due to the low number of samples for E1 yolk and J gill, those stages are not taken into account for statistics. The lines illustrate the shift of the haemocyanin isoforms.

is also present in SoH3. The translated sequence still contains $0.2 \%$ ambiguities (6 aa of 3,338 aa in total: FUa: 2, FUb: 2, FUc: 2), which all resemble isofunctional amino acid exchanges. The linker introns found between the FUs are all in phase 1. The internal introns in the signal peptide (phase 0), FUb (phase 2), FUe (phase 0) and FUf (phase 0) reflect the situation found in E. dofleini referring to location and phase [10], whereas the one found in FUg (phase 1) represents the internal intron also present in N. pompilius [6] (Figure 2). The haemocyanin protein SoH3 has a calculated molecular mass of 377,793 Da with a theoretical isoelectric point (pI) of 5.94. Each FU shows the characteristics for molluscan haemocyanin also found in Enteroctopus and Nautilus [6]

Table 2 Percentage of the different isoforms adding to haemocyanin mRNA

\begin{tabular}{cccc}
\hline Sample & SoH1 & SoH2 & SoH3 \\
\hline E1_Y & 0 & 0.001 & 99.999 \\
E1_E & 0 & 0.016 & 99.984 \\
E2_E & 0 & 0.249 & 99.751 \\
E3_E & 0.031 & 3.499 & 96.469 \\
E4_E & 1.657 & 31.73 & 66.613 \\
H_G & 9.674 & 90.062 & 0.264 \\
A_BG & 12.799 & 87.174 & 0.027 \\
\hline
\end{tabular}

The percentage is given for the respective tissues (Y: yolk; E: embryo; G: gill; $B G$ : branchial gland) of different ontogenetic stages ranging from embryos (E1-E4) to hatchlings $(\mathrm{H})$ and adults $(\mathrm{A})$.
(Figure 3). These are six histidine residues for copper binding (histidine residues in total: 173) as well as one thioether bridge (Cys73-His76) and three potential disulphide bridges (Cys62-Cys73, Cys187-Cys254, Cys344-Cys356), whereas the last one is not present in FUb and FUc. Ten potential glycosylation sites can be detected, nine NXT motifs and one NXS motif (Figure 3), whereas in FUc, FUd and FUe the central proline should block the glycosylation site [61]. The observed glycosylation pattern of SoH3 reflects the one already described for cuttlefish SoH1 [62] and cephalopods in general [6]: no glycosylation of FUc, one conserved glycosylation site (aa 415) in FUa, FUb, FUd, FUd' and FUe, one in FUg (aa 66) and another one present in FUd and FUd' (aa 268), which is probably not glycosylated in FUd' [62]. Differences concerning the glycosylation appear in FUe and FUf, in which SoH3 exposes more potential sites for glycosylation (FUe: aa 14 and aa 125, FUf: aa 163 and aa 402). The amino acid composition between the isoforms does not differ considerably.

\section{Phylogeny of cephalopod haemocyanin}

The amino acid sequences share identities of $81.7 \%$ for SoH1-SoH2, 78.5\% for SoH1-SoH3 and 77\% for SoH2SoH3. The topology of the maximum likelihood and the Bayesian trees are alike, therefore both results are displayed in one tree given both the support and bootstrap values, respectively. The phylogeny of cephalopod 


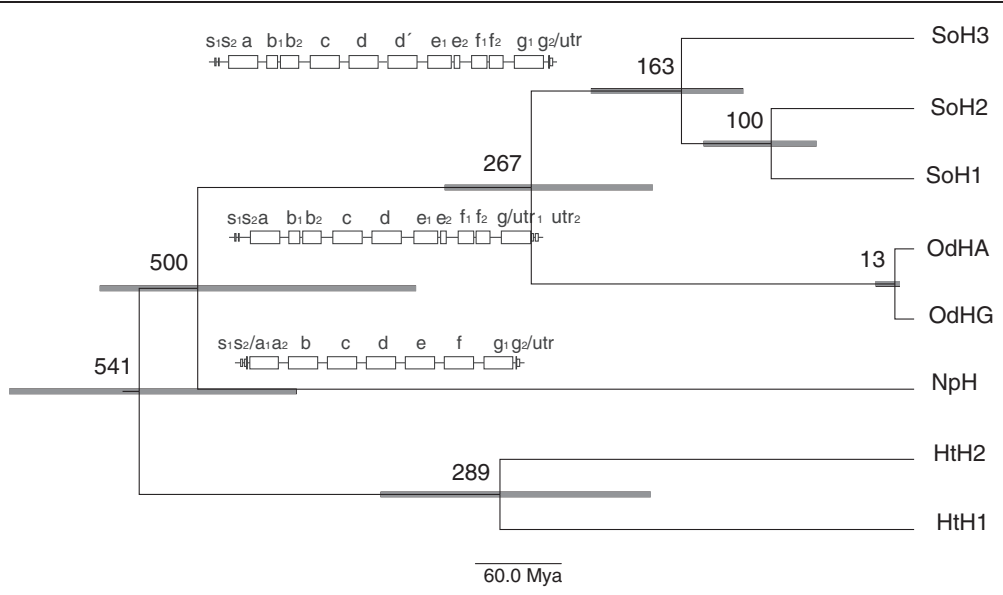

Figure 2 Molecular clock and exon-intron structure of cephalopod haemocyanin. Relaxed molecular clock with a mean evolutionary rate of $7.636 \times 10^{-4}$ calculated with BEAST v1.5.4 based on a maximum likelihood tree for the amino acid sequences of the complete haemocyanin molecules of Sepia officinalis ( $\mathrm{SoH} 1-\mathrm{SoH} 3)$, Enteroctopus dofleini (OdHA, OdHG), Nautilus pompilius ( $\mathrm{NpH}$ ) and Haliotis tuberculata ( $\mathrm{HtH} 1$, HtH2). The split of gastropoda and cephalopoda about $550 \pm 50$ Mya was used as calibration point. Included are the exon-intron structures of the haemocyanin of N. pompilius, E. dofleini and S. officinalis. Grey bars indicate the 95\% HPD (highest posterior density), i.e. the Bayesian confidence interval of the estimated age.

haemocyanin with the two isoforms of the gastropod Haliotis tuberculata as an outgroup is displayed in Figure 4.

Likewise, the phylogeny of FUd and FUd' is shown in Figure 5.

The relaxed molecular clock with a mean evolutionary rate of $7.636 \times 10^{-4}$ and a standard deviation of 0.237 revealed divergence times for the split of Tetrabranchiata-Dibranchiata of about 500 Mya and the split of Octobrachia-Decabrachia of about 267 Mya (Figure 2). Accordingly, the first duplication of cuttlefish haemocyanin occurred about 163 Mya and the second one about 100 Mya.

\section{Discussion}

\section{Haemocyanin during embryogenesis}

$\mathrm{SoH} 3$ is the most prominent haemocyanin isoform expressed in all analysed embryonic stages, whereas SoH1 and SoH2 expression starts during organogenesis (Lemaire 18-30). After hatching, the expression of SoH3 decreases drastically in the branchial gland (see also [22]). Hence, we could observe a shift of the haemocyanin isoforms during ontogeny. Accordingly, SoH3 represents an embryonic haemocyanin, whereas SoH1 and $\mathrm{SoH} 2$ are dominant in adult specimens. This is supported by the results of Decleir et al. $(1970,1971)$ $[47,48]$, who detected electrophoretically distinct protein fractions of cuttlefish haemocyanin during ontogeny. However, Ruth et al. (1999) [63] could not detect haemocyanin in sections of the midgut gland and branchial gland of Sepia embryos younger than NAEF stage XVII (representing E2 of this study) by immunohistochemistry. In contrast, Wolf et al. [64] found evidence for haemocyanin in extracts of eggs and embryos (larger than $0.5 \mathrm{~mm}$ in mantle length) using immunoelectrophoresis. In vertebrates, a developmental shift of the respiratory protein is well studied $[40,41]$ and also for invertebrates it has already been described $[31,37,38]$. Yet Sepia officinalis represents the only mollusc for which an embryonic form of haemocyanin has been published. Only a truncated haemocyanin form has so far been found in egg masses of Biomphalaria glabrata [65], a freshwater snail exhibiting haemoglobin as their respiratory protein. Additionally, we have some evidence for an embryonic haemocyanin isoform in the keyhole limpet Megathura crenulata according to protein analysis (Lieb et al., unpublished).

SoH3 mRNA was also detected in yolk sac samples of S. officinalis, which at least in some way compares to humans, where the formation of blood cells also starts in the yolk sac [66]. Yet, this raises the question about the site of synthesis of haemocyanin during early embryogenesis. In Cancer magister the haemocyanin found in oocytes is of maternal origin and is subsequently replaced by an embryonic one [33]. In the gastropod Haliotis asinina no maternal haemocyanin mRNA could be detected, but the expression of haemocyanin started $9 \mathrm{~h}$ after fertilisation [67]. Considering that SoH3 mRNA is present in the embryo until hatching, it might rather represent an embryonic than a maternal haemocyanin, as maternal mRNA also requires a maternal translation machinery and is usually essential during early embryogenesis, but starts to be degraded partly from egg activation on and partly from the onset of zygotic transcription [68]. Decleir et al. [48] proposed the production of embryonic haemocyanin of cuttlefish in the perivitelline membrane of the yolk sac. In different cephalopods this 


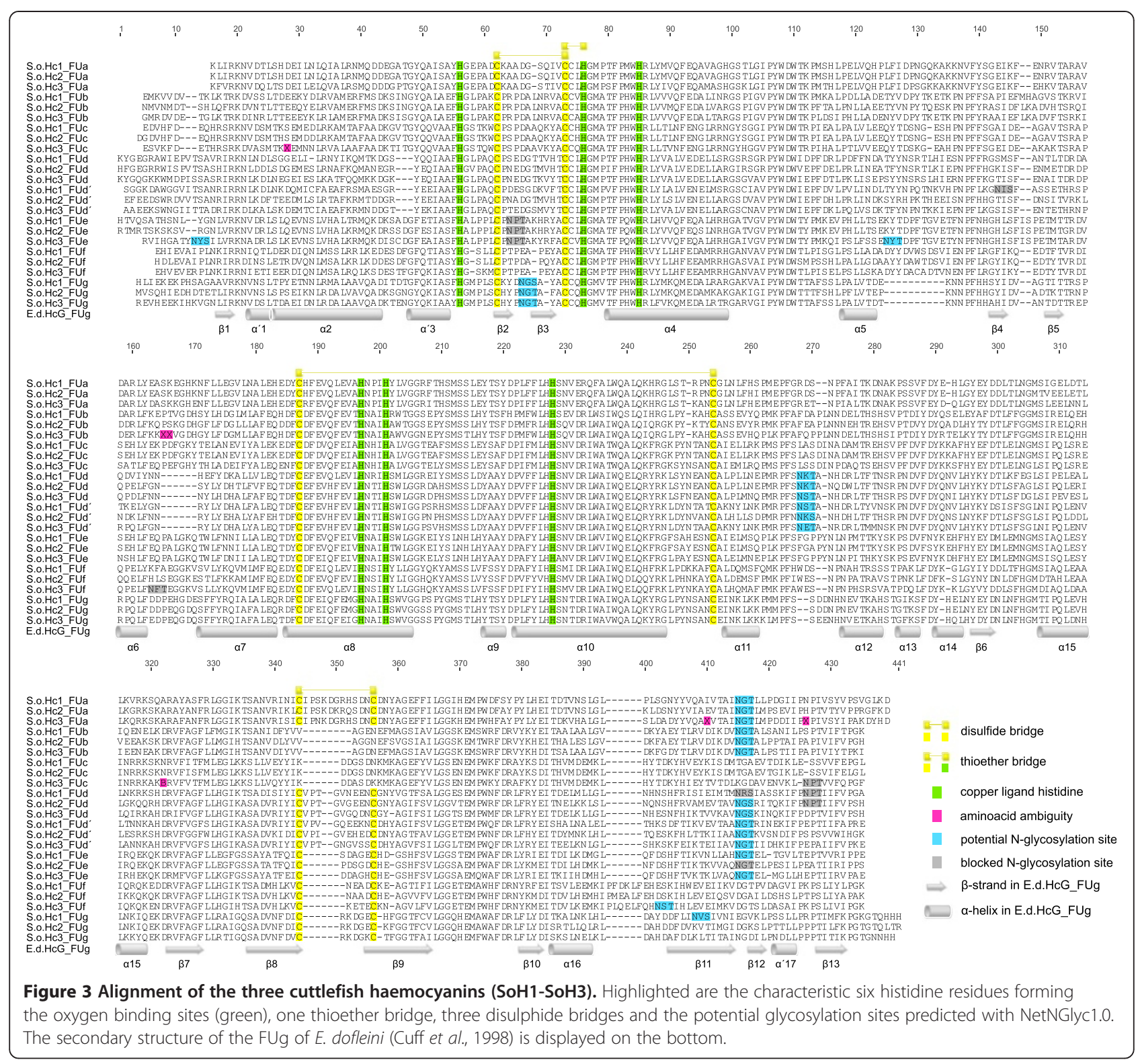

site was indeed described as an enzymatically very active one yet only with respect to yolk digestion [69]. A production of embryonic haemocyanin in the yolk sac could very well also be the case as copper, the metal ion complexing oxygen in the haemocyanin molecule, is found in the yolk sac during early development and is only later on transferred into the embryo, whereas the total amount of copper remains constant during embryogenesis [70]. Furthermore, the yolk sac may be favoured for oxygen uptake in the early stages because of its greater surface compared to the embryo [71].

The expression site of haemocyanin is not uniform among the Mollusca and ranges from the rhogocytes/ pore cells in the midgut gland in many gastropods [72-74] and Nautilus (a rather ancient cephalopod, which did not evolve branchial glands $[63,75])$, to the branchial glands in adult coleoid cephalopods. According to Beuerlein et al. [49] and Ruth et al. [75], the haemocyanin of cuttlefish embryos is also expressed in the midgut gland. This shift of the site of synthesis of haemocyanin during ontogeny may reflect an evolutionary shift from the midgut gland to the branchial gland in more highly evolved molluscs, namely recent coleoid cephalopods. The different expression patterns of the isoforms observed before hatching (E4) in the complete embryo (this study) and those observed only in gills (see [22]) may point to tissue differences in the expression and synthesis of the different isoforms at that developmental stage. Decleir et al. [48] proposed a shift of haemocyanin expression from the perivitelline membrane in early embryonic stages to the 


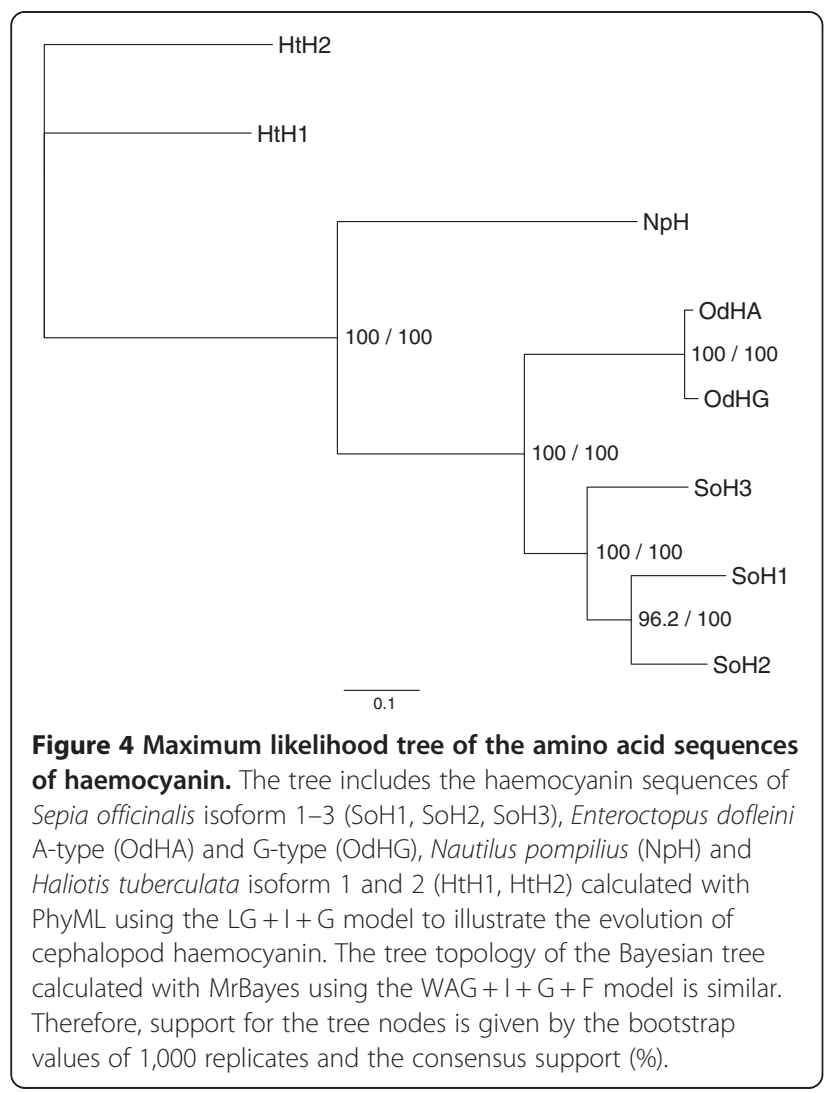

Figure 4 Maximum likelihood tree of the amino acid sequences of haemocyanin. The tree includes the haemocyanin sequences of Sepia officinalis isoform 1-3 (SoH1, SoH2, SoH3), Enteroctopus dofleini A-type (OdHA) and G-type (OdHG), Nautilus pompilius $(\mathrm{NpH})$ and Haliotis tuberculata isoform 1 and $2(\mathrm{HtH} 1, \mathrm{HtH} 2)$ calculated with PhyML using the $L G+I+G$ model to illustrate the evolution of cephalopod haemocyanin. The tree topology of the Bayesian tree calculated with MrBayes using the WAG $+I+G+F$ model is similar. Therefore, support for the tree nodes is given by the bootstrap midgut gland when both tissues get in close contact and eventually to the branchial glands when they are fully developed. This was supported by Beuerlein et al. [49] at the mRNA level and immunologically by Ruth et al. [63], who concluded that these tissues undergo a switch of function in haemocyanin metabolism during ontogeny. However, as none of these studies distinguished between the different haemocyanin isoforms, the different isoforms cannot be assigned to specific expression sites for the time being.

The sequence data indicate that $\mathrm{SoH} 3$, which is the first isoform to be expressed during ontogeny, resulted from the first gene duplication and could be closest to the common ancestor of cuttlefish haemocyanin (Figure 4). The isoforms SoH1 and SoH2 resulted from a second gene duplication. SoH1, whose expression starts last, may represent the most derived isoform referring to the number of substitutions per site. As the differences in substitutions per site between the cuttlefish haemocyanin isoforms are fairly small, this assumption is speculative. On the other hand, molluscan haemocyanin generally has a nearly constant and only moderate evolutionary rate; therefore large differences between isoforms are not to be expected. In this line of thought, the dominating haemocyanin isoform as well as the site of synthesis of haemocyanin could represent a rather ancient state during embryogenesis. This insinuates that the ontogeny of cuttlefish haemocyanin, i.e. the shift of composition and

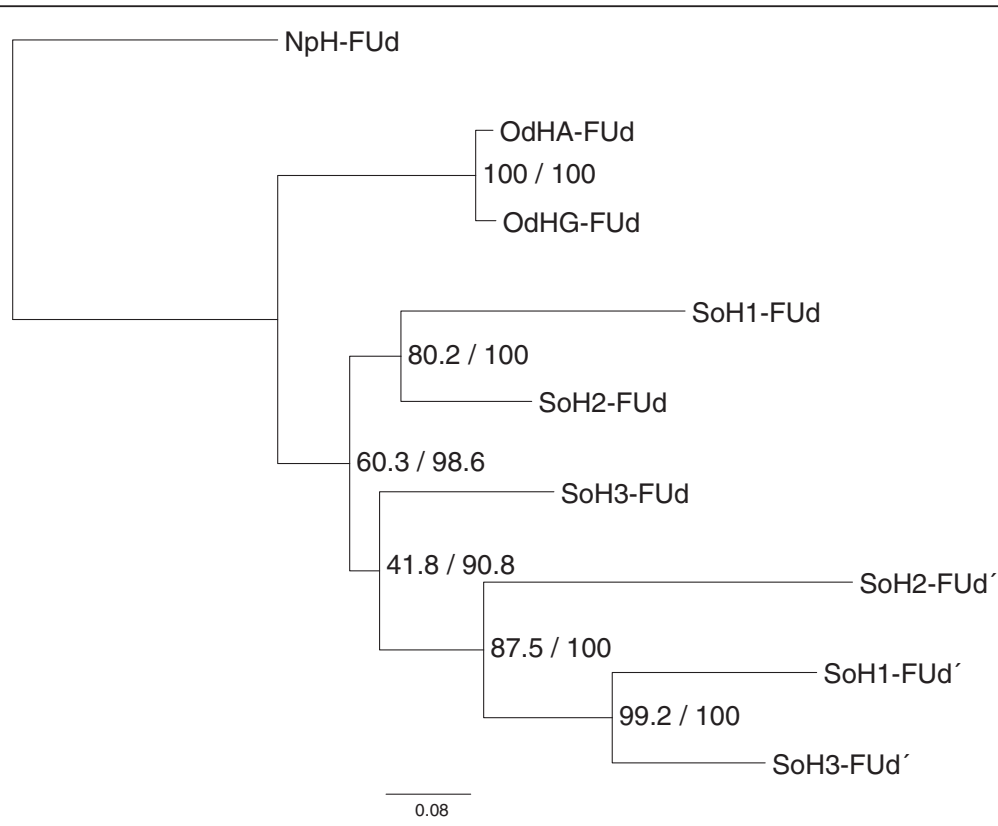

Figure 5 Maximum likelihood tree of the amino acid sequences of FUd and FUd'. The tree includes the FUd and FUd' sequences of Sepia officinalis (SoH1-SoH3), Enteroctopus dofleini (OdHA, OdHG) and Nautilus pompilius (NpH) and was calculated with PhyML using the WAG + I $+\mathrm{G}$ model to illustrate the duplication of FUd/d'. The tree topology of the Bayesian tree calculated with MrBayes using the WAG $+\mathrm{G}+\mathrm{F}$ model is similar. Therefore, support for the tree nodes is given by bootstrap values of 1,000 replicates and the consensus support (\%). 
expression site $[49,63]$, may recapitulate its phylogeny, which is in accordance with Haeckel's biogenetic law [76].

\section{Physiological aspects}

Although the timing for the completion of the final site of haemocyanin synthesis, the branchial glands, would be well in line with the start of expression of SoH1 and SoH2, the change in expression site does not explain the need for a special embryonic version of the respiratory protein. SoH3 could additionally exhibit physiological properties, which might suit the embryonic situation better. Crustacean and mammalian embryonic respiratory proteins for example show different oxygen affinities compared to their adult equivalents to cope with the challenges during embryogenesis [41,43] and shifts of haemocyanin function and expression have also been discussed for gastropods $[67,77]$. Cuttlefish embryogenesis is characterised by hypoxic and hypercapnic conditions in the perivitelline fluid (PVF) towards the end of development [44] resulting from the egg capsule acting as a diffusion barrier $[45,46]$. According to Gutowska and Melzner [44], the $\mathrm{PCO}_{2}$ of the PVF in eggs of Sepia officinalis increases during embryogenesis from $0.13 \mathrm{kPa}$ to $0.41 \mathrm{kPa}(0.04 \mathrm{kPa}$ in ambient seawater), corresponding to a decrease in $\mathrm{pH}$ from 7.72 to 7.23 .

The $\mathrm{O}_{2}$ affinity of cephalopod haemocyanin is strongly $\mathrm{pH}$-dependent [29] and could therefore be influenced by increasing carbon dioxide pressure $\left(\mathrm{PCO}_{2}\right)$ towards the end of embryogenesis, which is estimated to rise to 0.6$0.8 \mathrm{kPa}$ at the end of embryogenesis [44]. Furthermore, respiratory proteins of organisms living under hypoxia, i.e. the oxygen minimum zones (OMZ), express higher oxygen affinities in order to cope with the reduced oxygen availability $[78,79]$. The three haemocyanin isoforms of $S$. officinalis exhibit different theoretical isoelectric points (SoH1: 5.79, SoH2: 5.85, SoH3: 5.94) and different amounts of histidine residues (SoH1: 168, SoH2: 179, SoH3: 173) pointing at different buffer capacities and $\mathrm{O}_{2}$ affinities. These could come in handy during embryogenesis as abiotic conditions change, however clearly require further analysis before conclusions on the function of Hc3 can be reached.

\section{Phylogenetic aspects}

The embryonic haemocyanin of cuttlefish already represents the third isoform found in S. officinalis. It has the same genetic structure (presence of signal peptide, number of FUs, exon/intron structure) as found in the other isoforms. As mentioned above, it could also be the isoform most similar to the common ancestor of cuttlefish haemocyanin referring to the number of substitutions per site (Figure 4). The ambiguities that we could not clarify with the help of the genomic data may indicate SNPs between alleles. The unresolved positions observed in the cDNA sequence only could therefore be a result of pooled samples or even propose the existence of more than three isoforms, as fragments differing from all haemocyanin sequences found so far in the cuttlefish were also found at the genomic level.

Referring to the number of oxygen binding sites and the molecular weight of haemocyanin proteins, it has been suggested that Nautilus and Octopus exhibit 7 FUs whereas the haemocyanin of Sepia and Loligo consists of 8 FUs [1]. As molluscan haemocyanin evolved from subsequent gene duplications, an additional duplication could have occurred within the Dibranchiata. This is supported by molecular data for Spirula spirula and Sepia officinalis SoH1, SoH2 and now also SoH3, which indicate a duplication of FUd/d' [11,13] (DeGeest, unpublished, see also Figure 5) that is present in neither Nautilus nor Octopus [6,9]. It is rather unlikely that the duplication occurred independently in all four haemocyanins. Furthermore, the tree topology shown in Figure 5 supports the assumption that the FU duplication was prior to the isoform duplication in cuttlefish, as orthologues of both FUs (e.g. SoH1_FUd', SoH2_FUd', SoH3_FUd') and not paralogues (e.g. SoH1_FUd, SoH1_FUd') are assigned to each other. Referring to FUd, $\mathrm{SoH} 1$ is more closely related to $\mathrm{SoH} 2$, whereas FUd' indicates that SoH1 is more closely related to SoH3. These differences in topology between the two groups of orthologues resulting from different substitution rates also point to a duplication into FUd/d' much earlier than the duplication of the isoforms. This would support the assumption of FUd' being characteristic for Dibranchiata [13]. However, no sequence information for other groups within the Dibranchiata, e.g. squids, is available so far.

In contrast to the duplication into FUd/d' the duplication of the complete haemocyanin molecule occurred independently in several molluscs (Haliotis tuberculata [16,17], Megathura crenulata [19], Nucula nucleus [8], Enteroctopus dofleini [13], Sepia officinalis (DeGeest, unpublished)). Compared to the rather recent haemocyanin duplication in Enteroctopus about 13 Mya, the first duplication of the cuttlefish haemocyanin occurred about 163 Mya resulting in the ancestor of SoH3 (Figure 2). The second duplication about 100 Mya led to the progenitor of SoH1 and SoH2. These calculated divergence times correspond well to fossil data and previous results $[6,12,13]$.

\section{Conclusion}

The haemocyanin isoform 3 in cuttlefish represents an embryonic version of the respiratory pigment as it is expressed on a high level during embryogenesis, but is downregulated after hatching. Concerning its physiological properties only speculations based on the pI deduced from the primary structure can be done so far. 
The structural organisation of SoH3 with respect to the exon-intron structure and the duplication of FUd/ FUd' reflects the situation found in the other cuttlefish haemocyanin isoforms. Compared to these, SoH3 probably represents a rather ancient state with respect to its sequence and expression site, thereby leading to the assumption that phylogeny might be recapitulated during ontogeny.

\section{Abbreviations}

SoH1: Sepia officinalis haemocyanin isoform 1; SoH2: Sepia officinalis haemocyanin isoform 2; SoH3: Sepia officinalis haemocyanin isoform 3; OdHA: Enteroctopus dofleini haemocyanin type A; OdHG: Enteroctopus dofleini haemocyanin type $\mathrm{G}$; $\mathrm{HtH} 1$ : Haliotis tuberculata haemocyanin isoform 1; $\mathrm{HtH}$ 2: Haliotis tuberculata haemocyanin isoform 2; NpH: Nautilus pompilius haemocyanin; FU: Functional unit; SU: Subunit; Bp: Basepairs; Aa: Aminoacids; Mya: Million years ago; $\mathrm{PO}_{2}$ : Oxygen partial pressure; $P \mathrm{CO}_{2}$ : Carbondioxide partial pressure; UTR: Untranslated region; ORF: Open reading frame; COX: Cytochrome c oxidase; E1: Embryonic stage 1 (Lemaire 21-24); E2: Embryonic stage 2 (Lemaire 25-26); E3: Embryonic stage 3 (Lemaire 26-27); E4: Embryonic stage 4 (Lemaire 28-29); H: hatchling (Lemaire 30, hatchlings); A1: Adult stage.

\section{Competing interests}

The authors declare that they have no competing interests.

\section{Authors' contributions}

AT and FCM conceived and designed the study. AT, MO and BL participated in the sequencing, sequence analysis and/or interpretation. AT and FCM carried out the expression experiments and/or data analysis. AT drafted the manuscript, and FCM and BL revised it critically. All authors read and approved the final manuscript.

\section{Acknowledgements}

This work was kindly supported by the Deutsche Forschungsgemeinschaft DFG grants MA 4271/1-1\&2 to FCM. We are grateful to Dr. Folco Giomi for providing cuttlefish eggs and to Dr. Heidrun Windisch for her support in the expression data analysis.

\section{Author details}

${ }^{1}$ Integrative Ecophysiology, Alfred Wegener Institute for Polar and Marine Research, 27570 Bremerhaven, Germany. ${ }^{2}$ Institute of Zoology, Johannes Gutenberg University of Mainz, 55099 Mainz, Germany.

Received: 8 August 2013 Accepted: 20 November 2013

Published: 5 February 2014

\section{References}

1. Van Holde K, Miller Kl: Hemocyanins. Adv protein chem 1995, 47:1-81.

2. Burmester T: Molecular evolution of the arthropod hemocyanin superfamily. Mol Biol Evol 2001, 18(2):184-195.

3. Aguilera F, McDougall C, Degnan BM: Origin, evolution and classification of type-3 copper proteins: lineage-specific gene expansions and losses across the Metazoa. BMC Evol Biol 2013, 13(1):96.

4. Markl J: Evolution of Molluscan Hemocyanin Structures. Biochim Biophys Acta 2013, 1834(9):1840-1852.

5. Herskovits TT, Hamilton MG: Higher order assemblies of molluscan hemocyanins. Comp Biochem Physiol B 1991, 99(1):19.

6. Bergmann S, Lieb B, Ruth P, Markl J: The hemocyanin from a living fossil, the cephalopod Nautilus pompilius: protein structure, gene organization, and evolution. J Mol Evol 2006, 62(3):362-374.

7. Lieb $B$, Altenhein B, Markl J: The sequence of a gastropod hemocyanin ( $\mathrm{HtH} 1$ from Haliotis tuberculata). J Biol Chem 2000, 275(8):5675-5681.

8. Bergmann S, Markl J, Lieb B: The first complete CDNA sequence of the hemocyanin from a bivalve, the protobranch Nucula nucleus. $J \mathrm{Mol}$ Evol 2007, 64(5):500-510.

9. Miller Kl, Cuff ME, Lang WF, Varga-Weisz P, Field KG, Van Holde KE: Sequence of the Octopus dofleini hemocyanin subunit: structural and evolutionary implications1. J Mol Biol 1998, 278(4):827-842.
10. Lieb B, Altenhein B, Markl J, Vincent A, Van Olden E, Van Holde KE, Miller Kl: Structures of two molluscan hemocyanin genes: significance for gene evolution. Proc Natl Acad Sci USA 2001, 98(8):4546.

11. Lamy J, You V, Taveau JC, Boisset N, Lamy JN: Intramolecular localization of the functional units of Sepia officinalis hemocyanin by immunoelectron microscopy. J Mol Biol 1998, 284(4):1051-1074.

12. Lieb B, Markl J: Evolution of molluscan hemocyanins as deduced from DNA sequencing. Micron 2004, 35:117-119.

13. Warnke KM, Meyer A, Ebner B, Lieb B: Assessing divergence time of Spirulida and Sepiida (Cephalopoda) based on hemocyanin sequences. Mol Phylogenet Evol 2011, 58(2):390-394.

14. Lieb B, Boisguerin V, Gebauer W, Markl J: cDNA sequence, protein structure, and evolution of the single hemocyanin from Aplysia californica, an opisthobranch gastropod. J Mol Evol 2004, 59(4):536-545.

15. Mangum CP, Miller Kl, Scott SL, Van Holde KE, Morse MP: Bivalve hemocyanin: Structural, functional, and phylogenetic relationships. Biol Bull 1987, 173:205-221.

16. Keller H, Lieb B, Altenhein B, Gebauer D, Richter S, Stricker S, Markl J: Abalone (Haliotis tuberculata) hemocyanin type $1(\mathrm{HtH} 1)$. Eur J Biochem 1999, 264(1):27-38.

17. Altenhein B, Markl J, Lieb B: Gene structure and hemocyanin isoform $\mathrm{HtH} 2$ from the mollusc Haliotis tuberculata indicate early and late intron hot spots. Gene 2002, 301(1):53-60.

18. Swerdlow RD, Ebert RF, Lee P, Bonaventura C, Miller Kl: Keyhole limpet hemocyanin: structural and functional characterization of two different subunits and multimers. Comp Biochem Physiol B Biochem Mol Biol 1996, 113(3):537-548.

19. Gebauer W, Harris J, Heid H, Süling M, Hillenbrand R, Söhngen S, WegenerStrake A, Markl J: Quaternary structure, subunits and domain patterns of two discrete forms of keyhole limpet hemocyanin: KLH1 and KLH2. Zoology 1994, 98(1):51-68.

20. Gebauer W, Stoeva S, Voelter W, Dainese E, Salvato B, Beltramini M, Markl J: Hemocyanin subunit organization of the gastropod Rapana thomasiana. Arch Biochem Biophys 1999, 372(1):128-134.

21. Melzner F, Mark FC, Pörtner HO: Role of blood-oxygen transport in thermal tolerance of the cuttlefish, Sepia officinalis. Integr Comp Biol 2007. 47(4):645-655.

22. Strobel A, Hu MYA, Gutowska MA, Lieb B, Lucassen M, Melzner F, Pörtner $\mathrm{HO}$, Mark FC: Influence of temperature, hypercapnia, and development on the relative expression of different hemocyanin isoforms in the common cuttlefish Sepia officinalis. J Exp Zool A Ecol Genet Physiol 2012, 317A:511-523.

23. Andersen $\mathrm{O}$, Wetten OF, De Rosa MC, Andre C, Carelli Alinovi C, Colafranceschi M, Brix O, Colosimo A: Haemoglobin polymorphisms affect the oxygen-binding properties in Atlantic cod populations. Proc Royal Soc 2009, 276:833-841

24. Paul R, Zeiss B, Lamkemeyer T, Seidl M, Pirow R: Control of oxygen transport in the microcrustacean Daphnia: regulation of haemoglobin expression as central mechanism of adaptation to different oxygen and temperature conditions. Acta Physio/ Scand 2004, 182(3):259-275.

25. Zeiss B, Becher B, Lamkemeyer T, Rolf S, Pirow R, Paul RJ: The process of hypoxic induction of Daphnia magna hemoglobin: subunit composition and functional properties. Comp Biochem Physiol B Biochem Mol Biol 2003, 134(2):243-252.

26. Guadagnoli J, Braun A, Roberts S, Reiber C: Environmental hypoxia influences hemoglobin subunit composition in the branchiopod crustacean Triops longicaudatus. J Exp Biol 2005, 208(18):3543-3551.

27. Spicer J, El-Gamal M: Hypoxia accelerates the development of respiratory regulation in brine shrimp-but at a cost. J Exp Biol 1999, 202(24):3637-3646.

28. O'Dor R, Webber D: The constraints on cephalopods: why squid aren't fish. Can J Zool 1986, 64(8):1591-1605.

29. Brix O, Bårdgard A, Cau A, Colosimo A, Condo S, Giardina B: Oxygenbinding properties of cephalopod blood with special reference to environmental temperatures and ecological distribution. J Exp Zool 1989, 252(1):34-42.

30. Pörtner $\mathrm{H}-\mathrm{O}$ : An analysis of the effects of $\mathrm{pH}$ on oxygen binding by squid (Illex illecebrosus, Loligo pealei) haemocyanin. J Exp Biol 1990, 150(1):407-424.

31. Terwilliger NB, Ryan M: Ontogeny of crustacean respiratory proteins. Am Zool 2001, 41(5):1057-1067. 
32. Durstewitz G, Terwilliger NB: Developmental changes in hemocyanin expression in the Dungeness crab. Cancer magister. J Biol Chem 1997, 272(7):4347

33. Terwilliger NB, Dumler K: Ontogeny of decapod crustacean hemocyanin: effects of temperature and nutrition. J Exp Biol 2001, 204(5):1013-1020.

34. Wache S, Terwilliger NB, Terwilliger RC: Hemocyanin structure changes during early development of the crab Cancer productus. J Exp Zool 2005, 247(1):23-32.

35. Wache SC: Subunit structure of Cancer productus hemocyanin during early development, Phd thesis. Oregon: University of Oregon, Department of Biology; 1985

36. Heip J, Moens $L$, Joniau M, Konda M: Ontogenetical studies on extracellular hemoglobins of Artemia salina. Dev Biol 1978, 64:73-81.

37. Sánchez D, Ganfornina MD, Gutiérrez G, Bastiani MJ: Molecular characterization and phylogenetic relationships of a protein with potential oxygen-binding capabilities in the grasshopper embryo. A hemocyanin in insects? Mol Biol Evol 1998, 15(4):415-426.

38. Manwell C: Starch gel electrophoresis of the multiple haemoglobins of small and large larval Chironomus-a developmental haemoglobin sequence in an invertebrate. J Embryol Exp Morphol 1966, 16(2):259-270.

39. Vafopoulou-Mandalos $\mathrm{X}$, Laufer $\mathrm{H}$ : The ontogeny of multiple hemoglobins in Chironomus thummi (Diptera): The effects of a compound with juvenile hormone activity. Dev Biol 1982, 92(1):135-143.

40. Manwell C, Baker CMA, Betz TW: Ontogeny of haemoglobin in the chicken. J Embryol Exp Morphol 1966, 16(1):65-81.

41. Fantoni A, Farace M, Gambari R: Embryonic hemoglobins in man and other mammals. Blood 1981, 57(4):623-633.

42. Manning LR, Russell JE, Padovan JC, Chait BT, Popowicz A, Manning RS, Manning JM: Human embryonic, fetal, and adult hemoglobins have different subunit interface strengths. Correlation with lifespan in the red cell. Protein Sci 2007, 16(8):1641-1658.

43. Brown A, Terwilliger NB: Ontogeny of hemocyanin function in the Dungeness crab Cancer magister: Hemolymph modulation of hemocyanin oxygen-binding. J Exp Biol 1998, 201(6):819-826.

44. Gutowska MA, Melzner F: Abiotic conditions in cephalopod (Sepia officinalis) eggs: embryonic development at low pH and high $\mathrm{pCO}_{2}$ Mar Biol 2009, 156(3):515-519.

45. Cronin E, Seymour R: Respiration of the eggs of the giant cuttlefish Sepia apama. Mar Biol 2000, 136(5):863-870.

46. Wolf G, Verheyen E, Vlaeminck A, Lemaire J, Decleir W: Respiration of Sepia officinalis during embryonic and early juvenile life. Mar Biol 1985, 90(1):35-39.

47. Decleir W, Richard A: A study of the blood proteins in Sepia officinalis L. with special reference to embryonic hemocyanin. Comp Biochem Physiol 1970, 34(1):203-208. IN211, 209-211.

48. Decleir W, Lemaire J, Richard A: The differentiation of blood proteins during ontogeny in Sepia officinalis L. Comp Biochem Physiol B 1971, 40(4):923-928. IN925-IN926, 929-930.

49. Beuerlein K, Ruth P, Scholz FR, Springer J, Lieb B, Gebauer W, Westermann B, Schmidtberg H, Von Boletzky S, Markl J: Blood cells and the biosynthesis of hemocyanin in Sepia embryos. Micron 2004, 35(1-2):115-116.

50. Beuerlein K: Cytobiologische, immuncytochemische und molekularbiologische Untersuchungen zum Hämocyaninmetabolismus von Sepia officinalis $L$. (Cephalopoda), PhD Thesis. Giessen: Justus-Liebig-University Giessen; Department of Biology; 2002

51. Lemaire J: Table de développement embryonnaire de Sepia officinalis $\mathrm{L}$. (Mollusque Céphalopode). Bull Soc Zool Fr 1970, 95:773-782.

52. Hellemans J, Mortier G, De Paepe A, Speleman F, Vandesompele J: qBase relative quantification framework and software for management and automated analysis of real-time quantitative PCR data. Genome Biol 2007, 8(2):R19.

53. Vandesompele J, De Preter K, Pattyn F, Poppe B, Van Roy N, De Paepe A, Speleman F: Accurate normalization of real-time quantitative RT-PCR data by geometric averaging of multiple internal control genes. Genome Biol 2002, 3(7):R34

54. Noack S: Statistische Auswertung von Mess-und Versuchsdaten mit Taschenrechner und Tischcomputer: Anleitungen und Beispiele aus dem Laborbereich. Berlin: Walther de Gruyter; 1980.

55. The ExPASy Bioinformatics Resource Portal. http://web.expasy.org.
56. Talavera G, Castresana J: Improvement of phylogenies after removing divergent and ambiguously aligned blocks from protein sequence alignments. Syst Biol 2007, 56(4):564-577.

57. Darriba D, Taboada GL, Doallo R, Posada D: ProtTest 3: fast selection of best-fit models of protein evolution. Bioinformatics 2011, 27(8):1164-1165.

58. Huelsenbeck JP, Ronquist F: MRBAYES: Bayesian inference of phylogenetic trees. Bioinformatics 2001, 17(8):754-755

59. Guindon S, Gascuel O: A simple, fast, and accurate algorithm to estimate large phylogenies by maximum likelihood. Syst Biol 2003, 52(5):696-704.

60. Drummond AJ, Rambaut A: BEAST: Bayesian evolutionary analysis by sampling trees. BMC evolutionary biology 2007, 7(1):214

61. Bause E: Structural requirements of N-glycosylation of proteins. Studies with proline peptides as conformational probes. Biochem J 1983, 209(2):331

62. Gielens C, De Geest N, Compernolle F, Préaux G: Glycosylation sites of hemocyanins of Helix pomatia and Sepia officinalis. Micron 2004, 35(1):99-100.

63. Ruth P, Schimmelpfennig R, Schipp R: Comparative immunohistochemical and immunocytochemical investigations on the location of haemocyanin synthesis in dibranchiate and tetrabranchiate Cephalopods (Sepia and Nautilus). New York: Kluwer Academic/Plenum Publishers; 1999.

64. Wolf $\mathrm{G}$, Witters $\mathrm{R}$, Decleir W, Lontie R: Immunological evidence for haemocyanin-related proteins in mature eggs and embryos of Sepia officinalis L. Archs int Physiol Biochim 1980, 88:254.

65. Hathaway JJ, Adema CM, Stout BA, Mobarak CD, Loker ES: Identification of protein components of egg masses indicates parental investment in immunoprotection of offspring by Biomphalaria glabrata (Gastropoda, Mollusca). Dev Comp Immunol 2010, 34(4):425-435.

66. Tavian M, Péault B: Embryonic development of the human hematopoietic system. Int J Dev Biol 2005, 49(2-3):243-250.

67. Streit K, Jackson D, Degnan BM, Lieb B: Developmental expression of two Haliotis asinina hemocyanin isoforms. Differentiation 2005, 73(7):341-349.

68. Tadros W, Lipshitz HD: The maternal-to-zygotic transition: a play in two acts. Development 2009, 136(18):3033-3042.

69. Boletzky S: A contribution to the study of yolk absorption in the Cephalopoda. Zoomorphology 1975, 80(3):229-246.

70. Decleir W, Lemaire J, Richard A: Determination of copper in embryos and very young specimens of Sepia officinalis. Mar Biol 1970, 5(3):256-258.

71. Portmann A: Der embryonale Blutkreislauf und die Dotterresorption bei Loligo vulgaris. Zoomorphology 1926, 5(3):406-423

72. Albrecht U, Keller H, Gebauer W, Markl J: Rhogocytes (pore cells) as the site of hemocyanin biosynthesis in the marine gastropod Haliotis tuberculata. Cell Tissue Res 2001, 304(3):455-462.

73. Martin AM, Martin GG, Butler R, Goffredi SK: Synthesis of keyhole limpet hemocyanin by the rhogocytes of Megathura crenulata. Invert Biol 2011 , 130(4):302-312.

74. Manubens A, Salazar F, Haussmann D, Figueroa J, Del Campo M, Pinto JM, Huaquín L, Venegas A, Becker Ml: Concholepas hemocyanin biosynthesis takes place in the hepatopancreas, with hemocytes being involved in its metabolism. Cell Tissue Res 2010, 342(3):423-435.

75. Ruth P, Blum W, Bille J: Immunocytochemical reaction of a haemocyanin antibody in the midgut gland of Nautilus (Cephalopoda, Tetrabranchiata). Cell Mol Life Sci 1996, 52(6):549-553.

76. Haeckel EHPA: Generelle Morphologie der Organismen: allgemeine Grundzüge der organischen Formen-Wissenschaft begründert durch die von Charles Darwin reformierte Descendenz-Theorie vol. 2. Berlin: Georg Reimer; 1866.

77. Markl J, Savel-Niemann A, Wegener-Strake A, Süding M, Schneider A Gebauer W, Harris J: The role of two distinct subunit types in the architecture of keyhole limpet hemocyanin (KLH). Naturwissenschaften 1991, 78(11):512-514

78. Childress JJ, Seibel BA: Life at stable low oxygen levels: adaptations of animals to oceanic oxygen minimum layers. J Exp Biol 1998, 201(8):1223-1232.

79. Seibel BA: Critical oxygen levels and metabolic suppression in oceanic oxygen minimum zones. J Exp Biol 2011, 214(2):326-336

doi:10.1186/2041-9139-5-6

Cite this article as: Thonig et al:: A new haemocyanin in cuttlefish (Sepia officinalis) eggs: sequence analysis and relevance during ontogeny. EvoDevo 2014 5:6. 